\title{
ANALYSIS OF SHEET METAL FORMING OPERATIONS BY A STRESS RESULTANT CONSTITUTIVE LAW
}

\author{
C. H. CHOU AND J. PAN \\ 2250 G. G. Brown Building, Mechanical Engineering and Applied Mechanics, The University of Michigan, \\ Ann Arbor, MI 48109-2125, U.S.A. \\ S. C. TANG \\ Ford Motor Company, Dearborn, MI 48121, U.S.A.
}

\begin{abstract}
SUMMARY
Sheet metal forming is simulated by finite element methods using a stress resultant constitutive law in this paper. A Lagrangian description of axisymmetric and plane-strain shell deformation is first reviewed. Then a stress resultant constitutive law in rate form is presented, where the effect of thickness reduction due to large plastic deformation is considered. A finite element formulation in terms of stress resultants and their work-conjugate generalized strain rates is derived based on the virtual work principle. A hemispherical punch stretching operation and a plane-strain draw operation are simulated by a finite element program based on the finite element formulation. The results of these finite element simulations are in good agreement with those using the through-the-thickness integration method. The results of the hemispherical punch stretching simulation suggest that the coupling term of moments and membrane forces of the modified Ilyushin yield function should be eliminated to avoid numerical instability under stretching-dominated conditions for this rate-independent plasticity formulation. Further, the results suggest that the hardening rule in a power-law form based on the small-strain approach must be modified to take account for finite deformation effects of combined stretching and bending. Under the plane-strain draw operation, the sheet experiences a large amount of bending before the final stretching. The simulation based on the stress resultant constitutive law can produce this essential aspect of deformation pattern as that of the throughthe-thickness integration method, whereas a simulation based on a membrane theory cannot. In conclusion, the results of these simulations indicate that a finite element program based on the stress resultant constitutive law can simulate sheet-forming processes with much shorter computational time than that based on the through-the-thickness integration method.
\end{abstract}

\section{INTRODUCTION}

Many mathematical models have been proposed to simulate sheet metal forming processes from the viewpoints of materials, geometries and boundary conditions. The goal is to predict the formability of sheets and the punch forces of the processes. With the help of high-performance computers, we can now investigate the underlying physical phenomena in sheet metal forming processes by finite element methods. Different finite element models including membrane, solid and shell models have been proposed. The membrane model is the simplest one. However, the membrane model is not applicable where the bending of sheets becomes significant and dominant, for example, in drawing and binder wrap processes (for example, see References 1 and 2).

In general, the processes where bending becomes significant are simulated by the solid model or the shell model. For example, Wifi, ${ }^{3}$ Anderson ${ }^{4}$ and Makinouchi $^{5}$ adopted the solid model in their works, whereas Oñate and Zienkiewicz, ${ }^{6}$ Triantafyllidis and Samanta, ${ }^{7}$ Triantafyllidis 
et al., ${ }^{8}$ Wang and Tang ${ }^{1}$ and Lee et al. ${ }^{9}$ adopted the shell model. For the solid model, more elements are needed to describe these shell-type structures so that a large system of equations must be solved. For the shell model, although it is more effective than the solid model, it still takes a substantial amount of computational time and computer memory space for its three-dimensional calculation with integration in the thickness direction. Therefore, stress resultant constitutive laws have been proposed over the years to simulate the deformation of plate and shell structures under large plastic deformation in order to avoid the through-the-thickness integration to save computational time.

After Ilyushin ${ }^{10}$ developed the yield surface in the resultant space for perfectly plastic materials, many stress resultant constitutive laws have been developed to analyse the deformation and load-carrying capacity of plate and shell structures. For instances, based on the Tresca yield criterion, the limit loads of cylindrical shells were examined under axisymmetrical loading by Hodge $^{11}$ and Onat, ${ }^{12}$ and the elastic-plastic behaviour of cylindrical shells was examined by Brooks. ${ }^{13,14}$ Based on the von Mises yield criterion, the buckling phenomena of plates were investigated by Crisfield, ${ }^{15,16}$ and the elastic-plastic behaviour of shells was investigated under cyclic bending by Bieniek and Funaro; ${ }^{17}$ however, these research works do not include the effects of material strain hardening. With the material strain hardening included, the elastic-plastic behaviour of shells was analysed under cyclic bending and pre-loadings by Eidsheim and Larsen. ${ }^{18}$ Further applications of this type of theories are the analyses of viscoplastic shells, ${ }^{19}$ dynamically loaded plates, ${ }^{20}$ stiffened plates $^{21}$ and composite laminated plates. ${ }^{22}$ Also, the elastic-plastic behaviour of plates with a linear hardening was examined under a point load by Papadopoulos and Taylor, ${ }^{23}$ and the large deformation of a cylindrical panel problem was analysed by Morman et al. ${ }^{24}$

In this paper, we first review the kinematic relation between the displacements and generalized strains for a shell element under axisymmetric and plane-strain conditions based on the work of Wang and Tang. ${ }^{1}$ In their kinematic formulation, finite deformation effects are considered by keeping the high-order terms and using the true strain definition. Their kinematic formulation is based on the Kirchhoff assumption with consideration of thickness change. We adopt a recent stress resultant theory of Chou et al. ${ }^{25-27}$ where the hardening rule and modified Ilyushin yield surface of Chou et al. ${ }^{26}$ are constructed from the constitutive behaviour of power-law hardening materials within the context of the small-strain approach. Based on the virtual work principle, a finite element formulation in terms of stress resultants and the work-conjugate generalized strain rates is derived for finite deformation elastic-plastic problems. In the formulation, we neglect the high-order terms of the product of the out-of-plane co-ordinate and curvature in the integration through the thickness. The contact processes are simulated by the equilibrium equations of Tang, ${ }^{28}$ where both non-slip and slip contacts are considered. Also, in the finite element discretization process, the displacements are interpolated by Hermitian cubics. A Newton-Raphson scheme for numerical calculations to satisfy the virtual work principle is adopted in developing a finite element program.

Two sheet metal forming processes are investigated in this paper. The first is an axisymmetric punch stretching operation where a circular flat sheet is pressed by a dome-shaped punch through a circular arc-flanged die. The effect of a locked bead is considered by attaching a very stiff spring to the end of the sheet to control the flow of the sheet into the die. The results of the surface strains and punch forces are compared with those obtained from the through-the-thickness integration method based on the work of Wang and Tang. ${ }^{1}$ The second sheet metal forming process is a plane-strain draw operation where a sheet is allowed to draw into the die. This can be accomplished by placing a compliant spring at the end of the sheet. The sheet experiences a dominant bending and then unloading in contrast to that under the stretching operation. The 
results of the simulation are then compared with those obtained from the through-the-thickness integration method. Finally, the efficiencies of the computations based on the stress resultant theory and the through-the-thickness integration method are compared and discussed.

\section{DEFORMATION OF AXISYMMETRIC AND PLANE-STRAIN SHELL ELEMENTS}

A Lagrangian description is adopted here to describe the large deformation of a thin shell with reference to the undeformed flat configuration as shown in Figure 1. Denote $u$ and $w$ as the horizontal and vertical displacements, respectively, of a material point $x$ on the reference surface (i.e. the middle surface) of the undeformed flat configuration with the normal in the $\gamma$ direction. We assume that the normal of the shell middle plane remains normal after deformations. According to the work of Wang and Tang, ${ }^{1}$ the meridian and circumferential stretch ratios of an axisymmetric shell element, $\lambda_{1}$ and $\lambda_{2}$, at a normal distance $z$ away from the reference surface of the current (deformed) configuration are described, respectively, as

and

$$
\lambda_{1}=\lambda_{1}^{(0)}\left(1+\kappa_{1} z\right)
$$

$$
\lambda_{2}=\lambda_{2}^{(0)}\left(1+\kappa_{2} z\right)
$$

Here $\lambda_{1}^{(0)}$ and $\lambda_{2}^{(0)}$ are the stretch ratios of the reference surface in the meridian and circumferential directions, respectively. They can be expressed as

and

$$
\lambda_{1}^{(0)}=\left\{\left(1+u_{x}\right)^{2}+\omega_{x}^{2}\right)^{1 / 2}
$$

$$
\lambda_{2}^{(0)}=\frac{(x+u)}{x}
$$

where the subscript $x$ denotes the partial differentiation with respect to $x$. The principal curvatures of the reference surface in the meridian and circumferential directions are

and

$$
\kappa_{1}=\frac{w_{x} u_{x x}-\left(1+u_{x}\right) w_{x x}}{\left(\lambda_{1}^{(0)}\right)^{3}}
$$

$$
\kappa_{2}=-\frac{\omega_{x}}{(x+u) \lambda_{1}^{(0)}}
$$

where the subscript $x x$ represents the second partial derivative with respect to $x$.

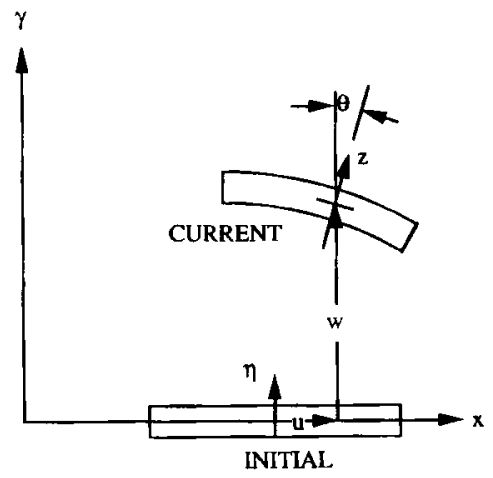

Figure 1. Initial and current configurations of a shell element 
The strain rates $\dot{\varepsilon}_{1}$ and $\dot{\varepsilon}_{2}$ are defined from the true strain definition as

$$
\dot{\varepsilon}_{\gamma}=\frac{\mathrm{d}\left(\ln \lambda_{\gamma}\right)}{\mathrm{d} t}=\frac{\dot{\lambda}_{\gamma}^{(0)}}{\lambda_{\gamma}^{(0)}}+\frac{z \dot{\kappa}_{\gamma}+\kappa_{\gamma} \dot{z}}{1+\kappa_{\gamma} z}
$$

where $\dot{\lambda}_{\gamma}^{(0)} / \lambda_{y}^{(0)}=\dot{e}_{\gamma}$ (no summation on $\gamma$ ) are the strain rates of the reference surface. In this paper, Greek subscripts range from 1 to 2 . With the assumption of the material incompressibility, the current distance $z$ of a material point from the reference surface can be related to its initial distance $\eta$ from the undeformed reference surface by

$$
\frac{\mathrm{d} z}{\mathrm{~d} \eta}=\left(\lambda_{1} \lambda_{2}\right)^{-1}
$$

Therefore, the initial distance $\eta$ from the middle surface can be expressed as a function of $z$, by integrating equation (4), as

$$
\eta=\lambda_{\nu}^{(0)} \lambda_{2}^{(0)}\left[z+\left(\kappa_{1}+\kappa_{2}\right) \frac{z^{2}}{2}+\kappa_{1} \kappa_{2} \frac{z^{3}}{3}\right]
$$

The rate $\dot{z}$ can be derived from the material incompressibility as

$$
\dot{z}=-\frac{z\left(1+\frac{\kappa_{1}+\kappa_{2}}{2} z+\frac{\kappa_{1} \kappa_{2}}{3} z^{2}\right)\left(\frac{\dot{\lambda}_{1}^{(0)}}{\lambda_{1}^{(0)}}+\frac{\dot{\lambda}_{2}^{(0)}}{\lambda_{2}^{(0)}}\right)+\left(\frac{z^{2}}{2}+\frac{\kappa_{2}}{3} z^{3}\right) \dot{\kappa}_{1}+\left(\frac{z^{2}}{2}+\frac{\kappa_{1}}{3} z^{3}\right) \dot{\kappa}_{2}}{\left(1+\kappa_{1} z\right)\left(1+\kappa_{2} z\right)}
$$

For plane-strain shell elements, the above relations are still valid but with $\lambda_{2}=1$ and $\kappa_{2}=0$.

\section{A STRESS RESULTANT CONSTITUTIVE LAW IN RATE FORM}

In an elastic-plastic analysis, a constitutive relation in rate form is usually needed because plastic deformation depends upon the prior deformation history. In Chou et al., ${ }^{27}$ a stress resultant constitutive law in rate form has been derived. We here reduce this rate form for axisymmetric and plane-strain applications. A convected co-ordinate is used for description of deformation. The $x-\gamma$ co-ordinate system as shown in Figure 1 is chosen such that the membrane shear forces and twisting moments vanish because of symmetry. The physical components of the stress resultant $\mathbf{S}$ referring to the deformed base vectors can be expressed as

$$
\mathbf{S}=\left\{N_{1}, N_{2}, M_{1}, M_{2}\right\}
$$

where $N_{1}$ and $N_{2}$ denote the membrane forces, and $M_{1}$ and $M_{2}$ the bending moments. The physical components of the work-conjugate generalized strain $\mathbf{E}$ referring to the deformed base vectors are

$$
\mathbf{E}=\left\{e_{1}, e_{2}, \kappa_{1}, \kappa_{2}\right\}
$$

where $e_{1}$ and $e_{2}$ denote the midplane strains and $\kappa_{1}$ and $\kappa_{2}$ the curvatures.

The physical components of the generalized strain rate $\dot{E}_{i}$ are assumed to be decomposed into the elastic parts $\dot{E}_{i}^{e}$ and the plastic parts $\dot{E}_{i}^{\text {p }}$,

$$
\dot{E}_{i}=\dot{E}_{i}^{\mathrm{e}}+\dot{E}_{i}^{\mathrm{p}}
$$

where the subscript $i$ range from 1 to 4 . It should be mentioned that the decomposition of the rate of deformation into additive elastic and plastic parts is generally true when the elastic strains are small (e.g. see Reference 29). We assume this additive decomposition for the generalized strain rate in equation (7) in order to maintain the simplicity and practical applicability of the theory for the range of the strains typically encountered in sheet metal forming applications. 
The physical components of the plastic generalized strain rate $\dot{E}_{i}^{\mathbf{p}}$ are defined, according to the associate flow rule, as

$$
\dot{E}_{i}^{\mathbf{p}}=\dot{\Lambda} \frac{\partial F}{\partial S_{i}}
$$

where $\dot{\Lambda}$ is a proportionality and $F$ is the yield function. The use of physical components in equations (7) and (8) is justified only when the deformed base vectors remain orthogonal. The yield function $F$ is assumed to be a linear function of the normalized stress resultant invariants $I_{N}, I_{M}$ and $I_{M N}:^{26}$

$$
F=\left\{I_{N}+\alpha I_{M}+\beta\left|I_{M N}\right|\right\}^{1 / 2}=\bar{\Sigma}
$$

where the parameters $\alpha$ and $\beta$ depend on the strain-hardening exponent $n$ of the uniaxial stress-strain relation from a tensile test. In equation (9), $\bar{\Sigma}$ represents the normalized equivalent stress resultant, and $I_{N}, I_{M}$ and $I_{M N}$ are the second-order invariants of the normalized stress resultants (without shear and twisting contributions). These invariants are expressed as

and

$$
\begin{gathered}
I_{N}=\bar{N}_{1}^{2}+\bar{N}_{2}^{2}-\bar{N}_{1} \bar{N}_{2} \\
I_{M}=\bar{M}_{1}^{2}+\bar{M}_{2}^{2}-\bar{M}_{1} \bar{M}_{2}
\end{gathered}
$$

$$
I_{M N}=\bar{N}_{1} \bar{M}_{1}+\bar{N}_{2} \bar{M}_{2}-\frac{1}{2} \bar{N}_{1} \bar{M}_{2}-\frac{1}{2} \bar{N}_{2} \bar{M}_{1}
$$

where $\bar{N}_{1}=N_{1} / \sigma_{0} h, \bar{N}_{2}=N_{2} / \sigma_{0} h, \bar{M}_{1}=4 M_{1} / \sigma_{0} h^{2}$ and $\bar{M}_{2}=4 M_{2} / \sigma_{0} h^{2}$. In these expressions, $\sigma_{0}$ is the initial yield stress and $h$ is the current plate thickness.

The physical components of the stress resultant rate $\mathbf{S}$ and the elastic generalized strain rate $\mathbf{E}^{\mathrm{e}}$ are assumed to be related via the usual elastic modulus $\mathbf{D}^{\mathrm{e}}$ as

$$
\dot{S}_{i}=D_{i j}^{\mathrm{e}} \dot{E}_{j}^{\mathrm{e}}=D_{i j}^{\mathrm{e}}\left(\dot{E}_{j}-\dot{E}_{j}^{\mathrm{p}}\right)
$$

where the subscripts $i$ and $j$ range from 1 to 4 . The physical components of the elastic modulus $\mathbf{D}^{\mathrm{e}}$ referring to the deformed base vectors are assumed as

$$
\left[\mathbf{D}^{\mathrm{e}}\right]=\frac{E h}{1-v^{2}}\left(\begin{array}{cccc}
1 & v & 0 & 0 \\
v & 1 & 0 & 0 \\
0 & 0 & \frac{h^{2}}{12} & \frac{v h^{2}}{12} \\
0 & 0 & \frac{v h^{2}}{12} & \frac{h^{2}}{12}
\end{array}\right)
$$

where $E$ is Young's modulus and $v$ is Poisson's ratio. It should be noted that a convected co-ordinate is used in our kinematic formulation. The deformed base vectors are orthogonal to each other and exhibit no in-plane rotation under axisymmetric and plane-strain deformation. Under these conditions we can use the time rate instead of the co-rotational rate of the physical components of $\mathbf{S}$ based on the deformed base vectors in equation (10).

The hardening rule for power-law hardening materials, as proposed in Chou et al., ${ }^{26}$ is

$$
\bar{\Sigma}=\bar{\Lambda}^{1 / n}
$$

where $n$ is the hardening exponent of the uniaxial stress-strain relation from a tensile test, and $\bar{\Lambda}$ is the normalized equivalent plastic generalized strain. Here $\bar{\Lambda}$ is accumulated over the deformation 
history as

$$
\bar{\Lambda}=\int_{0}^{t} \dot{\bar{\Lambda}} \mathrm{d} t
$$

where $t$ represents time. The relation between $\dot{\bar{\Lambda}}$ and $\dot{\Lambda}$ is $\dot{\bar{\Lambda}}=\dot{\Lambda} E /\left(\sigma_{0}^{2} h\right)$. Here $\dot{\bar{\Lambda}}$ is expressed as

$$
\dot{\bar{\Lambda}}=\left[\frac{4}{3}\left(I_{\dot{e}}+\frac{9}{16 \alpha} I_{\dot{\kappa}}-\frac{3 \beta \lambda}{8 \alpha} I_{\dot{e} \dot{\kappa}}\right)\right]^{1 / 2}
$$

where $I_{\dot{e}}, I_{\dot{\kappa}}$ and $I_{\dot{e} \dot{\kappa}}$ are the second-order invariants of the normalized plastic generalized strain rates. These invariants are expressed as

and

$$
\begin{aligned}
& I_{\dot{e}}=\dot{\bar{e}}_{1}^{\mathrm{p}^{2}}+\dot{\bar{e}}_{2}^{\mathrm{p} 2}+\dot{\bar{e}}_{1}^{\mathrm{p}} \dot{\bar{e}}_{2}^{\mathrm{p}} \\
& I_{\dot{\kappa}}=\dot{\bar{\kappa}}_{1}^{\mathrm{p}^{2}}+\dot{\bar{\kappa}}_{2}^{\mathrm{p} 2}+\dot{\bar{\kappa}}_{1}^{\mathrm{p}} \dot{\bar{\kappa}}_{2}^{\mathrm{p}}
\end{aligned}
$$

$$
I_{\dot{e} \dot{\kappa}}=2 \dot{\bar{e}}_{1}^{\mathrm{p}} \dot{\bar{\kappa}}_{1}^{\mathrm{p}}+2 \dot{\bar{e}}_{2}^{\mathrm{p}} \dot{\bar{\kappa}}_{2}^{\mathrm{p}}+\dot{\bar{e}}_{2}^{\mathrm{p}} \dot{\bar{\kappa}}_{1}^{\mathrm{p}}+\dot{\bar{e}}_{1}^{\mathrm{p}} \dot{\bar{\kappa}}_{2}^{\mathrm{p}}
$$

where $\dot{\bar{e}}_{1}=\dot{e}_{1} E / \sigma_{0}, \dot{\bar{e}}_{2}=\dot{e}_{2} E / \sigma_{0}, \dot{\bar{\kappa}}_{1}=\dot{\kappa}_{1} E h / 3 \sigma_{0}$ and $\dot{\bar{\kappa}}_{2}=\dot{\kappa}_{2} E h / 3 \sigma_{0}$.

Next, the consistency condition dictates the stress resultant state during the plastic flow to remain on the yield surface in the stress resultant space. Under this condition, we have

$$
\frac{\partial F}{\partial S_{i}} \dot{S}_{i}+\frac{\partial F}{\partial h} \dot{h}=\frac{\partial \bar{\Sigma}}{\partial \Lambda} \dot{\Lambda}
$$

where $\dot{h}=-\left(\dot{E}_{1}+\dot{E}_{2}\right) h$ is assumed. Multiplying equation (10) by $\partial F / \partial S_{i}$ and combining with equations (8) and (14) give the proportionality for plastic flow rate

$$
\dot{\Lambda}=\frac{\frac{\partial F}{\partial S_{m}} D_{m n}^{\mathrm{e}} \dot{E}_{n}-\frac{\partial F}{\partial h}\left(\dot{E}_{1}+\dot{E}_{2}\right) h}{\frac{\partial \bar{\Sigma}}{\partial \Lambda}+\frac{\partial F}{\partial S_{k}} D_{k l}^{\mathrm{e}} \frac{\partial F}{\partial S_{l}}}
$$

where the subscripts $k, l, m$ and $n$ range from 1 to 4 .

The rate form of the stress resultant constitutive law is derived by substituting equation (15) into equations (8) and (10) as

$$
\dot{S}_{i}=D_{i j}^{\text {ep }} \dot{E}_{j}
$$

In equation (16), the tangent moduli $D_{i j}^{\text {ep }}$ are defined as

$$
D_{i j}^{\mathrm{ep}}=D_{i k}^{\mathrm{e}}\left(I_{k j}-\frac{\frac{\partial F}{\partial S_{k}}\left(\frac{\partial F}{\partial S_{l}} D_{l j}^{\mathrm{e}}-\frac{\partial F}{\partial h}\left(\delta_{1 j}+\delta_{2 j}\right) h\right)}{\frac{\partial \bar{\Sigma}}{\partial \Lambda}+\frac{\partial F}{\partial S_{m}} D_{m n}^{\mathrm{e}} \frac{\partial F}{\partial S_{n}}}\right)
$$

where $I_{i j}$ are the elements of the unit matrix. In equation (17), $\partial \bar{\Sigma} / \partial \Lambda$ can be written as

$$
\frac{\partial \bar{\Sigma}}{\partial \Lambda}=\frac{\partial \bar{\Sigma}}{\partial \bar{\Lambda}} \frac{\partial \bar{\Lambda}}{\partial \Lambda}=\frac{E}{n \sigma_{0}^{2} h} \bar{\Sigma}^{1-n}
$$


Also, $\partial F / \partial S_{i}$ and $\partial F / \partial h$ can be derived from the yield function in equation (9) as

$$
\begin{aligned}
& \frac{\partial F}{\partial S_{1}}=\frac{1}{\sigma_{0}^{2} \bar{\Sigma}}\left\{\frac{1}{h^{2}}\left(2 N_{1}-N_{2}\right)+\frac{4 \beta \xi}{h^{3}}\left(M_{1}-\frac{M_{2}}{2}\right)\right\} \\
& \frac{\partial F}{\partial S_{2}}=\frac{1}{\sigma_{0}^{2} \bar{\Sigma}}\left\{\frac{1}{h^{2}}\left(2 N_{2}-N_{1}\right)+\frac{4 \beta \xi}{h^{3}}\left(M_{2}-\frac{M_{1}}{2}\right)\right\} \\
& \frac{\partial F}{\partial S_{3}}=\frac{1}{\sigma_{0}^{2} \bar{\Sigma}}\left\{\frac{16 \alpha}{h^{4}}\left(2 M_{1}-M_{2}\right)+\frac{4 \beta \xi}{h^{3}}\left(N_{1}-\frac{N_{2}}{2}\right)\right\} \\
& \frac{\partial F}{\partial S_{4}}=\frac{1}{\sigma_{0}^{2} \bar{\Sigma}}\left\{\frac{16 \alpha}{h^{4}}\left(2 M_{2}-M_{1}\right)+\frac{4 \beta \xi}{h^{3}}\left(N_{2}-\frac{N_{1}}{2}\right)\right\}
\end{aligned}
$$

and

$$
\frac{\partial F}{\partial h}=-\frac{1}{\bar{\Sigma}}\left\{\frac{2}{h} I_{N}+\alpha \frac{4}{h} I_{M}+\beta \frac{3}{h}\left|I_{M N}\right|\right\}
$$

where $\xi=\operatorname{sgn}\left(I_{M N}\right)\left(\xi=1\right.$ when $I_{M N}>0$ and $\xi=-1$ when $\left.I_{M N}<0\right)$.

Within the context of the small-strain approach, the undeformed geometry is treated as the reference and is assumed not to change. Therefore, in the hardening rule of the small-strain theory, $h$ is the initial thickness and treated as a constant. However, we will use the current thickness as the input to the normalized hardening rule of equation (11) for our finite-strain formulation here. It should be noted that the hardening rule in equation (11) is the exact one for the von Mises materials under pure membrane forces when $h$ is taken as the current thickness within the finite deformation approach.

\section{FINITE ELEMENT FORMULATION}

The virtual work principle in terms of the physical components of the stress measure and the strain rate measure for axisymmetric problems can be derived ${ }^{30,31,1}$ as

$$
\iint_{-h_{0} / 2}^{h_{0} / 2}\left(\sigma_{1} \delta \dot{\varepsilon}_{1}+\sigma_{2} \delta \dot{\varepsilon}_{2}\right) \mathrm{d} \eta \mathrm{d} A_{0}=\int\left(f_{1} \delta \dot{u}+f_{3} \delta \dot{w}\right) \mathrm{d} A
$$

where $\sigma_{1}$ and $\sigma_{2}$ are the principal Kirchhoff stresses in the meridian and circumferential directions, respectively, $h_{0}$ is the initial sheet thickness, $\mathrm{d} A_{0}$ denotes the differential area in the initial configuration, $\mathrm{d} A$ denotes the differential surface area in the deformed configuration, and $f_{1}$ and $f_{3}$ are the current surface load components in the $x$ and $\gamma$ directions, respectively. From the virtual work principle in equation (19) and the kinematic relation in equations (3)-(6), the virtual work principle in terms of stress resultants can be derived by integration through the thickness.

In sheet metal forming operations, the thin-shell assumption is generally true for the sheet when the thickness is small compared with the radius of curvature. The high-order terms of $\kappa_{x} z$ due to the contribution of $\dot{z}$ are therefore neglected because of the thin-shell assumption. The virtual work principle in terms of the physical components of the stress resultants and the generalized strain rates is expressed as

$$
\begin{aligned}
& \int\left\{\left(N_{1}-M_{2} \kappa_{2}\right) \delta \dot{e}_{1}+\left(N_{2}-M_{1} \kappa_{1}\right) \delta \dot{e}_{2}+M_{1} \delta \dot{\kappa}_{1}+M_{2} \delta \dot{\kappa}_{2}\right\} \lambda_{1}^{(0)} \lambda_{2}^{(0)} \mathrm{d} A_{0} \\
& =\int\left(f_{1} \delta \dot{u}+f_{3} \delta \dot{w}\right) \mathrm{d} A
\end{aligned}
$$


where $N_{\gamma}$ and $M_{\gamma}$ are the principal membrane forces and bending moments referring to the current configuration, $e_{\gamma}$ are the midplane strains, and $\kappa_{\gamma}$ are the curvatures. Note that the material incompressibility is assumed in the derivation of equation (20).

The stiffness equations in rate form can be derived using equation (20) and the constitutive relation in equation (16). As in the work of Wang and Tang, ${ }^{1}$ Hermitian cubic polynomials are used as the interpolation functions for $u$ and $w$ and three-point Simpson's rule is used for numerical integration over the element domain $\mathrm{d} A_{0}$. The nodal velocity vector $\dot{U}$ of the $i$ th node is arranged as

$$
\dot{\mathbf{U}}^{(i) \mathrm{T}}=\left(\dot{u}^{(i)}, \dot{u}_{x}^{(i)}, \dot{w}^{(i)}, \dot{w}_{x}^{(i)}\right)
$$

Then the resulting stiffness matrix equation is expressed as

$$
\mathbf{K} \dot{\mathbf{U}}=\dot{\mathbf{F}}
$$

where $\overrightarrow{\mathbf{F}}$ is the nodal force rate vector from equation (20). The contribution from the $i$ th element to the stiffness matrix $K$ in equation (22) can be written as

$$
\mathbf{K}^{(\mathbf{E}(i))}=\int \mathbf{N}^{\mathrm{T}}\left[\mathbf{H}^{\mathrm{T}} \mathbf{Q} \mathbf{H}+\left(N_{1}-M_{2} \kappa_{2}\right) \mathbf{a}+\left(N_{2}-M_{1} \kappa_{1}\right) \mathbf{b}+M_{1} \mathbf{c}+M_{2} \mathbf{d}\right] \mathbf{N d} A_{0}
$$

where $\mathbf{N}$ is a $(6 \times 8)$ element interpolation matrix defined as

$$
\dot{\mathbf{u}}=\mathbf{N} \dot{\mathbf{u}}^{(E(I))}
$$

In the above equation $\dot{\mathbf{u}}$ and $\dot{\mathbf{u}}^{(E(I))}$ are defined as

and

$$
\dot{\mathbf{u}}^{\mathrm{T}}=\left\{\dot{u}, \dot{w}, \dot{u}_{x}, \dot{w}_{x}, \dot{u}_{x x}, \dot{w}_{x x}\right\}
$$

$$
\dot{\mathbf{u}}^{(E(I))}=\left(\dot{\mathbf{U}}^{(i) \mathbf{T}}, \dot{\mathbf{U}}^{(i+1) \mathrm{T}}\right) \text {. }
$$

In equation (23), $\mathrm{H}$ is a $(4 \times 6)$ strain matrix

$$
\mathbf{H}=\left(\mathbf{g}^{\mathbf{T}}, \mathbf{h}^{\mathrm{T}}, \mathbf{p}^{\mathbf{T}}, \mathbf{q}^{\mathbf{T}}\right)^{\mathbf{T}}
$$

where $\mathbf{g}^{\mathbf{T}}, \mathbf{h}^{\mathbf{T}}, \mathbf{p}^{\mathbf{T}}$ and $\mathbf{q}^{\mathbf{T}}$ are listed in Wang and Tang. ${ }^{1}$ In equation (23), $\mathbf{Q}$ is a $(4 \times 4)$ matrix

$$
[Q]=\left(\begin{array}{cccc}
1 & 0 & 0 & \kappa_{2} \\
0 & 1 & \kappa_{1} & 0 \\
0 & 0 & 1 & 0 \\
0 & 0 & 0 & 1
\end{array}\right) D^{\text {ep }}+\left(\begin{array}{cccc}
N_{1}-M_{2} \kappa_{2} & N_{1}-M_{2} \kappa_{2} & 0 & -M_{2} \\
N_{2}-M_{1} \kappa_{1} & N_{2}-M_{1} \kappa_{1} & -M_{1} & \\
M_{1} & M_{1} & 0 & 0 \\
M_{2} & M_{2} & 0 & 0
\end{array}\right)
$$

where $D^{\mathbf{c p}}$ is the elastic-plastic stiffness matrix in equation (17). Also, a, b, $\mathbf{c}$ and $\mathbf{d}$ in equation (23) are the $(6 \times 6)$ symmetric matrices derived by Wang and Tang. ${ }^{1}$

We follow the method of Tang ${ }^{28}$ to satisfy the contact constraint and equilibrium check. Note that the external moments at any contact node are set to be zero. The above derivations are for the axisymmetric case. Under plane strain conditions, equations (19)-(23) remain valid with $\lambda_{2}=1$ and $\kappa_{2}=0$.

\section{A HEMISPHERICAL PUNCH STRETCHING SIMULATION}

Based on the standard procedure of finite element methods, the formulations in Sections 2-4 are employed in a finite element program to simulate a hemispherical punch stretching operation as schematically shown in Figure 2. The hemispherical (axisymmetric) punch stretching operation 


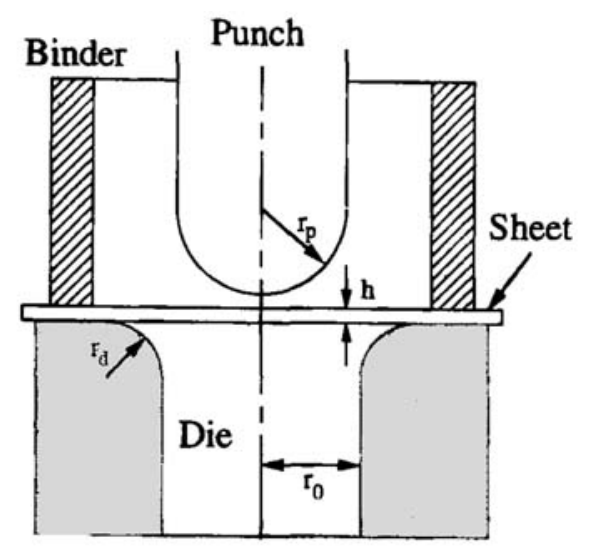

Figure 2. A hemispherical punch stretching operation

includes a punch, a die, a binder, and a piece of sheet metal. The geometric parameters of the punch stretching operation are $r_{\mathrm{p}}$ (the punch radius) $=50.8 \mathrm{~mm}, r_{\mathrm{d}}$ (the die profile radius) $=6.35 \mathrm{~mm}, r_{0}$ (the punch opening radius) $=52.83 \mathrm{~mm}$, and $h$ (the sheet thickness) $=1.2 \mathrm{~mm}$. The elastic material properties of the sheet metal are $E=206 \mathrm{GPa}$ and $v=0.3$. The uniaxial tensile stress-strain relationship for the sheet metal is taken as

$$
\begin{aligned}
& \sigma=E \varepsilon \text { for } \sigma \leqslant \sigma_{0} \\
& \sigma=K \varepsilon^{1 / n} \quad \text { for } \sigma \geqslant \sigma_{0}
\end{aligned}
$$

where $n$ is the hardening exponent, $\sigma_{0}$ is the reference yield stress, and $K$ represents a reference stress. Here we take $\sigma_{0}=103 \mathrm{MPa}$ and $n=4 \cdot 76$. For the given stress-strain relation, equation (18) should be modified as

$$
\frac{\partial \bar{\Sigma}}{\partial \Lambda}=\frac{E}{\sigma_{0}^{2} h} \frac{1}{n \bar{\Sigma}^{n-1}-1}
$$

The value of $\mu$ in Coulomb's friction law for contact surfaces is assumed to be $0 \cdot 17 .^{1}$ The equivalent spring constant $K_{\mathrm{b}}$ for the binder resistance is set at $1 \mathrm{GN} / \mathrm{mm}$ which is very large to simulate the stretching operation.

In order to investigate the accuracy and computational efficiency, the numerical results of the simulation based on stress resultants are compared with those based on the through-thethickness integration method. For conveniences of presentation, the computation based on stress resultants is referred to as the two-dimensional (2-D) computation and the computation based on the through-the-thickness integration method is referred to as the three-dimensional (3-D) computation.

When the modified Ilyushin yield function in equation (9) is adopted, the computation becomes divergent when the punch travel distance $d$ reaches $20.9 \mathrm{~mm}$. The distributions of the bottom surface strains are not smooth just before the divergence and these numerical results are presented in Chou. ${ }^{32}$ At this punch travel distance, we find that these elements under stretching-dominated conditions experience unloading and then reloading. This unstable numerical phenomenon possibly originates from the vertex existing on the yield surface along the pure stretching direction.

To remove the numerical instability due to the vertex, we neglect the coupling term $I_{M N}$ by setting $\beta=0$ in the modified Ilyushin yield function. We refer to this yield function with $\beta=0$ as 


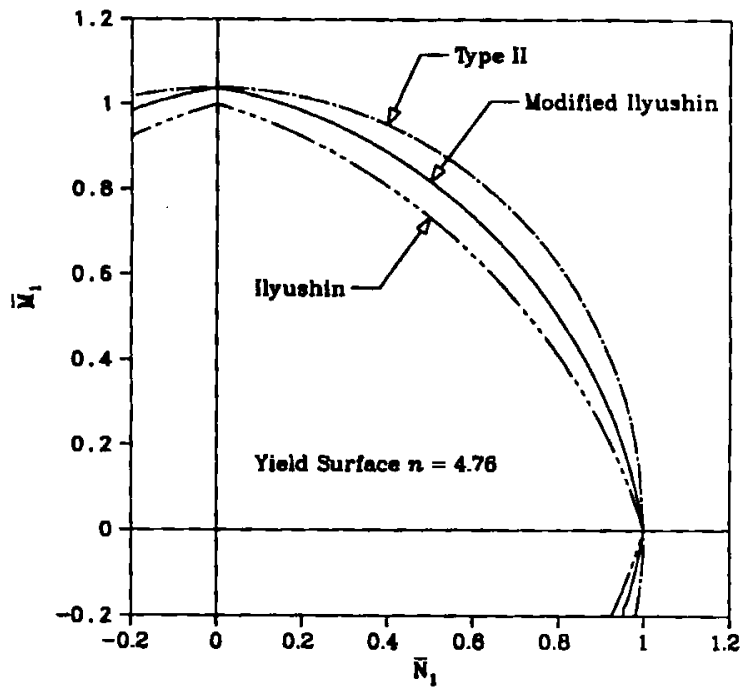

Figure 3. A comparison of the modified Ilyushin yield surface, the Type II yield surface, and the Ilyushin yield surface in the $\bar{N}_{1}-\bar{M}_{1}$ plane.

the Type II yield function. Figure 3 shows the modified Ilyushin and Type II yield surfaces for $n=4.76$ and the original Ilyushin yield surface in the $\bar{N}_{1}-\bar{M}_{1}$ plane. As shown in the figure, the size of the Type II yield surface is larger than that of the modified Ilyushin yield surface under combined bending and membrane force conditions. It should be mentioned that the original Ilyushin yield surface is for perfectly plastic materials and can be expressed by equation (9) with $\alpha=1$ and $\beta=1 / \sqrt{3}$. However, for power-law hardening materials $\alpha$ and $\beta$ in equation (9) depend upon the hardening exponent. In the normalized co-ordinate system shown in Figure 3 , the original Ilyushin yield surface appears to be smaller in size under pure bending and combined bending and membrane force conditions than the others. In other words, when the original Ilyushin yield function is used to describe the evolution of the yield surface in the stress resultant space, the bending capability of the plate or shell element is possibly underestimated under pure bending and combined bending and membrane force conditions.

Figure 4 shows the distributions of the bottom surface strains of the 3-D computation and the 2-D computation based on the Type II yield function at the punch travel distances $d$ of $8,16,24$ and $32 \mathrm{~mm}$. Note that the bottom surface strain is the most critical strain measure for this type of problems because it includes the contributions from both the membrane stretching and bending in our shell formulation. A comparison shows that the Type II yield function gives very good results for the punch distance $d$ up to $24 \mathrm{~mm}$, where the maximum radial strain becomes very large at about 16 per cent. In this type of sheet forming processes, the central area of the punch contacts the sheet first, and then the contact region expands. As the contact region expands, combined bending and stretching deformation gradually changes to stretching-dominated deformation. The contact surface is conditionally restricted to slip due to the frictional constraints. This means that the slip is restricted in the contact area and the area just outside the contact area must be stretched more to satisfy the geometric requirement. Therefore, the position of the maximum radial strain gradually moves out as the contact area expands.

At $d=32 \mathrm{~mm}$, the trends of the distributions of the bottom surface strains of the 2-D computation are the same as those of the 3-D computation. However, the maximum bottom 

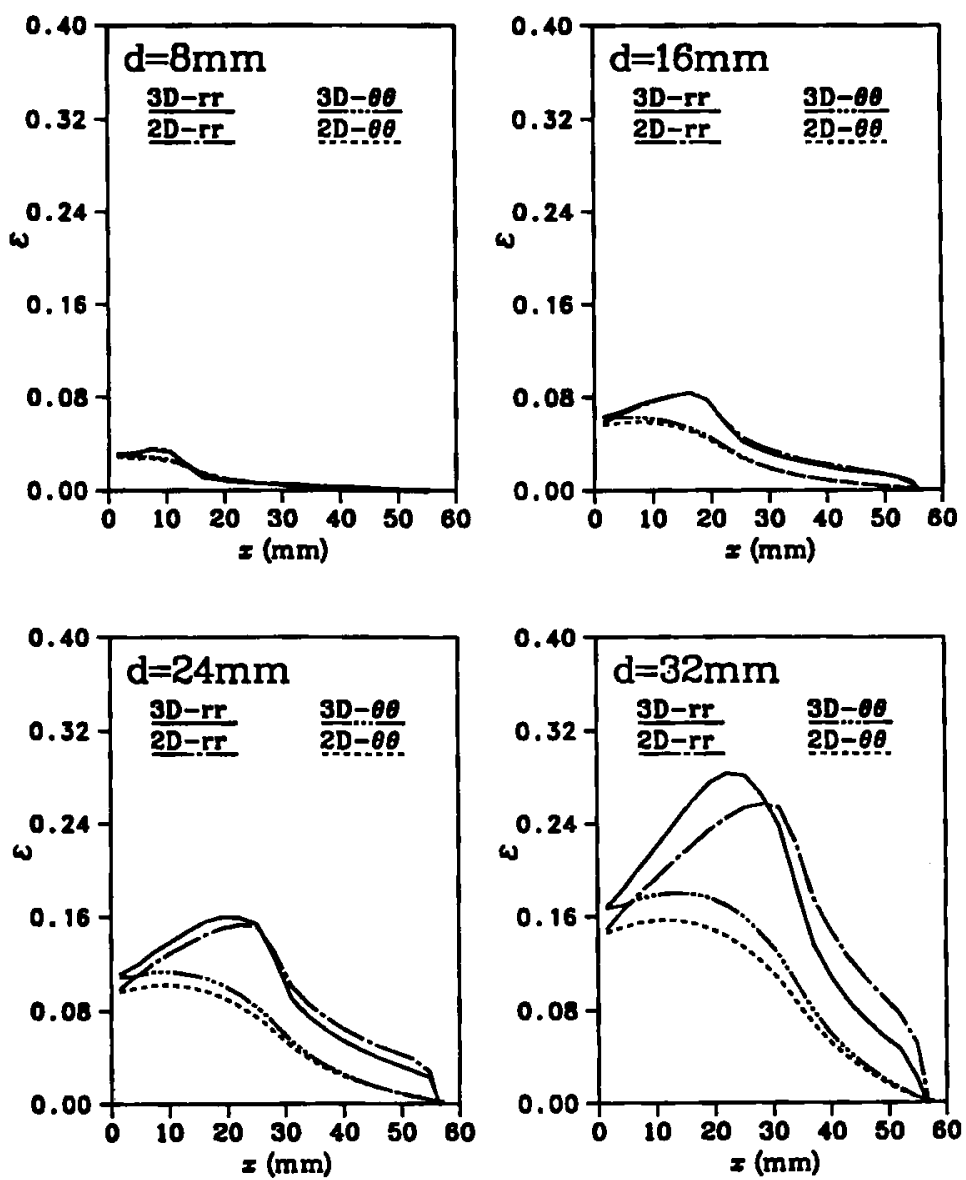

Figure 4. A comparison of the distributions of the bottom surface strains of the 3-D computation and the 2-D computation based on the Type II yield function for the hemispherical punch stretching operation

surface strain of the 3-D computation becomes larger than that of the 2-D computation. As we continue to increase the punch travel distance, the maximum strains of both the 2-D and the 3-D computations accelerate quickly to very large values where the necking failure should occur. This kind of localization phenomenon has been discussed by Triantafyllidis and co-workers. ${ }^{7,8}$ The strain localization phenomenon occurs at a much larger punch travel distance for the 2-D computation than that of the 3-D computation. The reason is that the hardening rule of the stress resultant theory is obtained with the small-strain assumption. The hardening rule becomes stiffer than that of the finite deformation formulation (Chou et $a l^{27}$ ) at large generalized strains. Due to the stiffer hardening rule, the punch force of the 2-D computation at large punch travel distances is larger than that of the 3-D computation as shown in Figure 5.

In order to improve the results of simulations, the hardening rule in equation (11) is modified to become more compliant at large strains by multiplying a parameter $\eta_{1}$ in an ad hoc manner according to the investigation in Chou et al. ${ }^{27}$ Now the modified hardening rule is expressed as

where

$$
\begin{gathered}
\Sigma=\eta_{1} \bar{\Lambda}^{1 / n} \\
\eta_{1}=1
\end{gathered}
$$




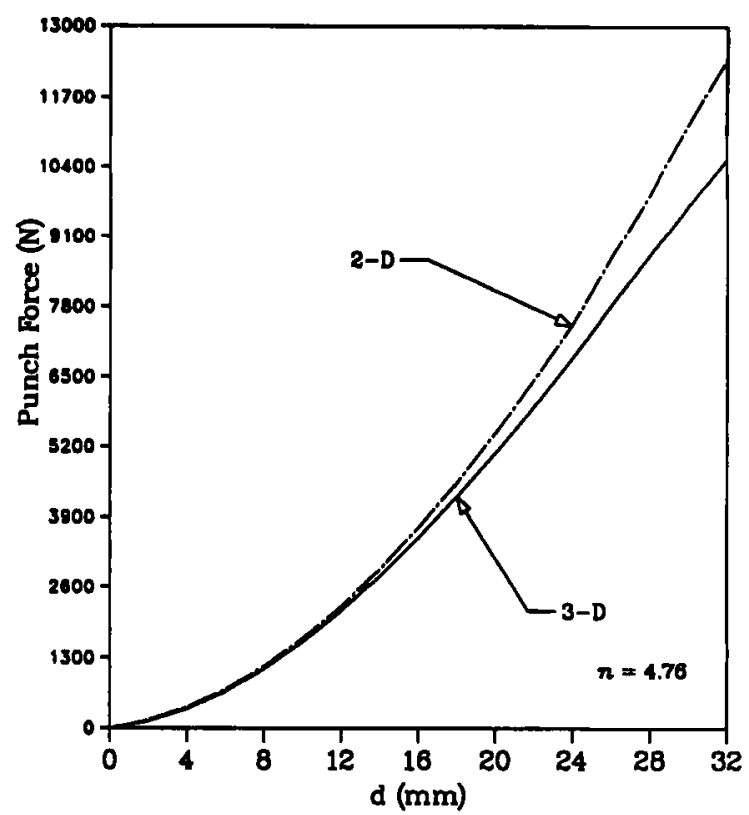

Figure 5. A comparison of the punch forces of the 3-D computation and the 2-D computation based on the Type II yield function for the hemispherical punch stretching operation

Table I. A comparison of the results of the 3-D and 2-D computations at the punch travel distance of 24 and $32 \mathrm{~mm}$ in a hemispherical punch stretching case

\begin{tabular}{|c|c|c|c|c|c|}
\hline $\begin{array}{l}d \\
(\mathrm{~mm})\end{array}$ & $\mu$ & Items & 2-D & 3-D & $\begin{array}{c}\text { Error or time } \\
\text { saving }(\%)\end{array}$ \\
\hline 24 & 0.17 & $\begin{array}{c}\text { Maximum } \varepsilon_{r r} \\
\text { Punch force } \\
\text { CPU time }\end{array}$ & $\begin{array}{c}0.153 \\
7455 \mathrm{~N} \\
9.96 \mathrm{~min}\end{array}$ & $\begin{array}{c}0.160 \\
6832 \mathrm{~N} \\
31.62 \mathrm{~min}\end{array}$ & $\begin{array}{r}4 \cdot 4 \\
9 \cdot 1 \\
68 \cdot 5\end{array}$ \\
\hline 32 & $0 \cdot 17$ & $\begin{array}{l}\text { Maximum } \varepsilon_{r r} \\
\text { Punch force } \\
\text { CPU time }\end{array}$ & $\begin{array}{c}0 \cdot 256 \\
12419 \mathrm{~N} \\
13 \cdot 28 \mathrm{~min}\end{array}$ & $\begin{array}{c}0.283 \\
10540 \mathrm{~N} \\
41.89 \mathrm{~min}\end{array}$ & $\begin{array}{c}9 \cdot 54 \\
17 \cdot 8 \\
68 \cdot 3\end{array}$ \\
\hline
\end{tabular}

when $\bar{\Lambda} \leqslant 100$, and

when $\bar{\Lambda}>100$.

$$
\eta_{1}=\frac{1}{4\left(1-\mathrm{e}^{-(\bar{\Lambda}-100) / 100}\right)+1}
$$

Figure 6 shows the distributions of the bottom surface strains of the 3-D computation and the 2-D computation based on the Type II yield function and the modified hardening rule. Figure 7 shows the punch forces as functions of the punch travel distances for the 3-D computation and the 2-D computation based on the Type II yield function and the modified hardening rule. As expected, the results of the 2-D computation with the modified hardening rule agree well with those of the 3-D computation.

Tables I and II summarize the results of the maximum bottom surface radial strain $\varepsilon_{r r}$, the punch force, and the CPU time for both the 2-D and the 3-D computations. These computations 

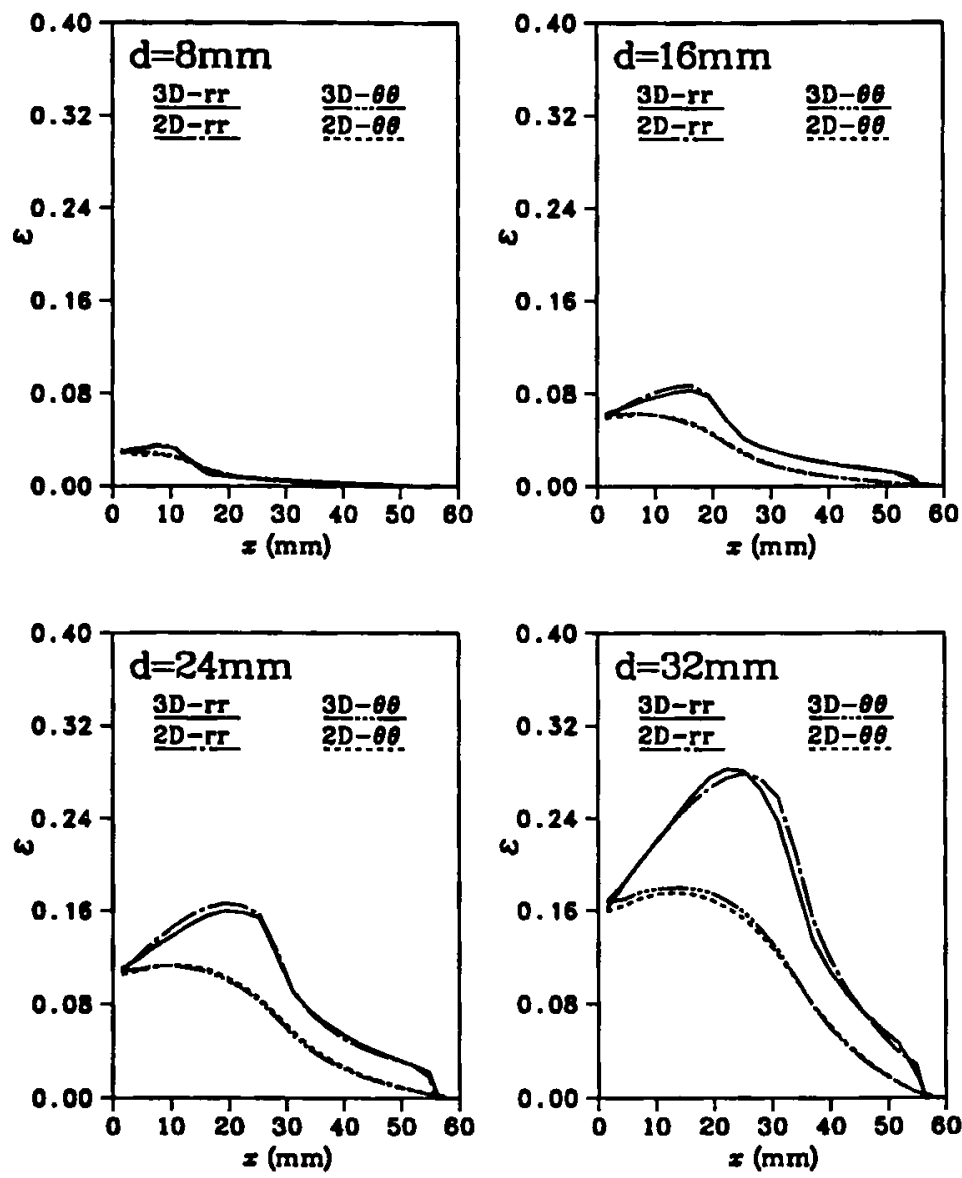

Figure 6. A comparison of the distributions of the bottom surface strains of the 3-D computation and the 2-D computation based on the Type II yield function and the modified hardening rule for the hemispherical punch stretching operation

Table II. A comparison of the results of the 3-D computation and the 2-D computation based on a modified hardening rule at the punch travel distance of $32 \mathrm{~mm}$ in a hemispherical punch stretching case

\begin{tabular}{|c|c|c|c|c|c|}
\hline $\begin{array}{l}d \\
(\mathrm{~mm})\end{array}$ & $\mu$ & Items & 2-D & 3-D & $\begin{array}{l}\text { Error or time } \\
\text { saving }(\%)\end{array}$ \\
\hline 24 & 0.17 & $\begin{array}{c}\text { Maximum } \varepsilon_{r r} \\
\text { Punch force } \\
\text { CPU time }\end{array}$ & $\begin{array}{c}0.166 \\
6838 \mathrm{~N} \\
10 \cdot 02 \mathrm{~min}\end{array}$ & $\begin{array}{c}0 \cdot 160 \\
6832 \mathrm{~N} \\
31 \cdot 62 \mathrm{~min}\end{array}$ & $\begin{array}{c}3 \cdot 75 \\
0.08 \\
68 \cdot 3\end{array}$ \\
\hline 32 & 0.17 & $\begin{array}{l}\text { Maximum } \varepsilon_{r r} \\
\text { Punch force } \\
\text { CPU time }\end{array}$ & $\begin{array}{c}0.279 \\
10894 \mathrm{~N} \\
13.68 \mathrm{~min} .\end{array}$ & $\begin{array}{c}0.283 \\
10540 \mathrm{~N} \\
41.89 \mathrm{~min}\end{array}$ & $\begin{array}{l}1 \cdot 41 \\
3 \cdot 36 \\
67 \cdot 3\end{array}$ \\
\hline
\end{tabular}




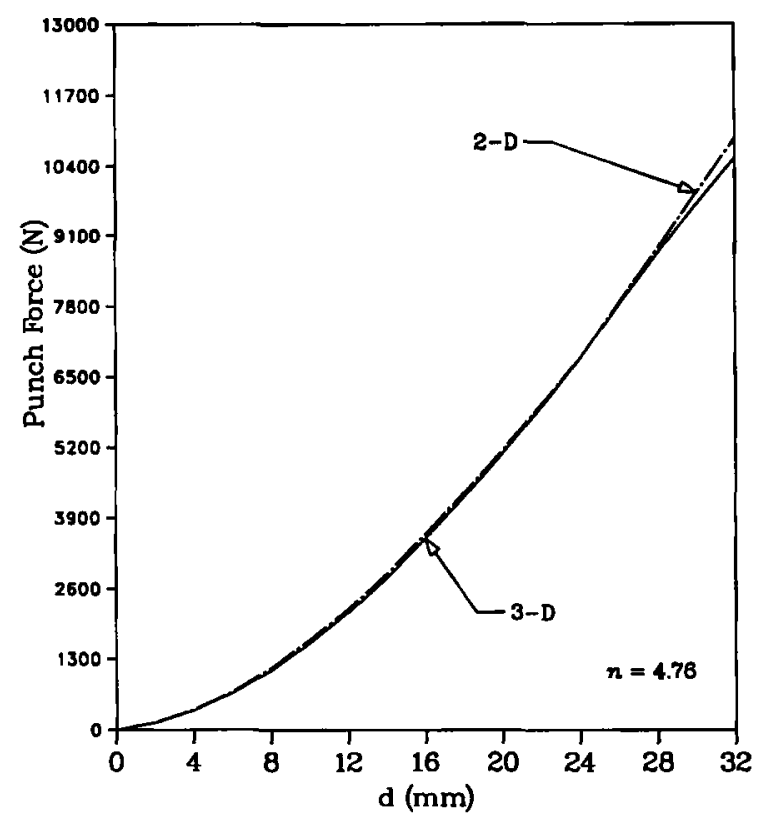

Figure 7. A comparison of the punch forces of the 3-D computation and the 2-D computation based on the Type II yield function and the modified hardening rule for the hemispherical punch stretching operation

were carried out on a VAX-8650 computer of Ford Motor Company. The results of the 2-D computation listed in Table I are based on the power-law hardening rule. The results of the 3-D computation are taken as the benchmarks to assess the error and saving of computational time of the 2-D computation. In Table $I$, at the punch travel distance $d=24 \mathrm{~mm}$, the errors of the maximum radial strain and the punch force of the 2-D computation are 4.4 and 9.1 per cent, respectively. These errors are relatively small in engineering applications. The reason for these errors is possibly due to the deviation of the direction of the outward normal to the Type II yield surface from that of the complementary potential surface of Chou et al. ${ }^{25}$ A parametric study of the direction of the outward normal to the Type II yield surface in the $\bar{N}_{1}-\bar{M}_{1}$ plane indicates that the direction near the $\bar{N}_{1}$ axis deviates to the $\bar{N}_{1}$ direction when compared with that of the complementary potential surface of Chou et al. ${ }^{25}$ Most importantly, the computational time of the 2-D computation is about 32 per cent of that of the 3-D computation. At $d=32 \mathrm{~mm}$, we still have the same computational time saving for the 2-D computation. However, the error of the maximum radial strain becomes large at about 10 per cent, and the error of the punch force increases to about 18 per cent. The reason for these large errors is that the 3-D computation generates localization (or necking) earlier than the 2-D computation since the hardening rule of the stress resultant theory is stiffer.

Table II shows a comparison of the 2-D results based on the modified hardening rule with those of the 3-D computation at $d=24$ and $32 \mathrm{~mm}$. The results of the 2-D computation are very close to those of the 3-D computation. For example, the largest error of the maximum radial strain is about 4 per cent at $d=24 \mathrm{~mm}$, and the largest error of the punch force is about 4 per cent at $d=32 \mathrm{~mm}$. The time-saving advantage is still the same. 


\section{A PLANE-STRAIN DRAW SIMULATION}

A plane-strain draw operation is schematically shown in Figure 8. The plane-strain draw operation includes a punch, a die, a binder and a piece of sheet metal. The geometric parameters of the plane-strain draw operation are $r_{\mathrm{p}}$ (the punch profile radius) $=5.5 \mathrm{~mm}, r_{\mathrm{d}}$ (the die profile radius) $=10.5 \mathrm{~mm}, w$ (the punch width) $=10.5 \mathrm{~mm}$ and $h$ (the sheet thickness) $=1.0 \mathrm{~mm}$. The material parameters of the sheet are $E=206.8 \mathrm{GPa}, v=0.3, \sigma_{0}=106.07 \mathrm{MPa}$ and $n=5$. This case is considered as well-lubricated and the values of $\mu$ in Coulomb's friction law is assumed to be 0.02 . The limit force of the equivalent elastic-plastic spring for the binder resistance is set at $250 \mathrm{~N} / \mathrm{mm}$ to simulate the draw operation. This operation is simulated to assess the applicability of the stress resultant theory under bending dominated and unloading conditions.

Figure 9 shows the distributions of the thickness strains of the 3-D computation and the 2-D computations based on both the modified Ilyushin and Type II yield function at the punch travel distance $d=20 \mathrm{~mm}$. There are three locations where the thickness reduction becomes localized due to bending operations. The 2-D computations based on the stress resultants give the exact locations and the approximate magnitudes for the thickness strains at these locations. This localization of thickness reduction cannot be obtained from the simulation based on the membrane theory. ${ }^{1}$ The results based on the membrane theory are shown as the horizontal line in Figure 9 for comparison. Also, there is no significant difference between the 2-D results based on the Type II and modified Ilyushin yield function.

Figure 10 shows the distributions of the bottom surface strains of the 3-D computation and the 2-D computation based on both the modified Ilyushin and Type II yield function at the punch travel distance $d=20 \mathrm{~mm}$. The results of the 2-D computations are very close to those of the 3-D computation regardless of using the modified Ilyushin yield function or the Type II yield function. As indicated in Chou et al., ${ }^{27}$ the stress resultant theory gives a very good prediction of plastic deformation of a plate element when the plastic strain is moderately larger than the elastic strain. However, when plastic deformation becomes very large, finite deformation effects must be considered. Then the hardening rule must be modified in the stress resultant approach as in the hemispherical punch stretching case. For this draw operation, the maximum plastic strain is not

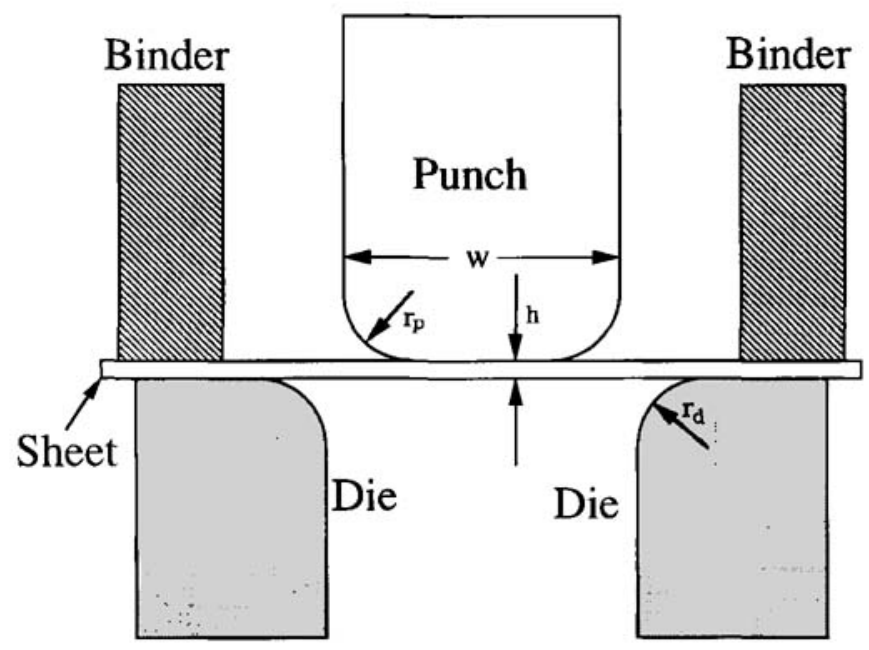

Figure 8. A plane-strain draw operation 

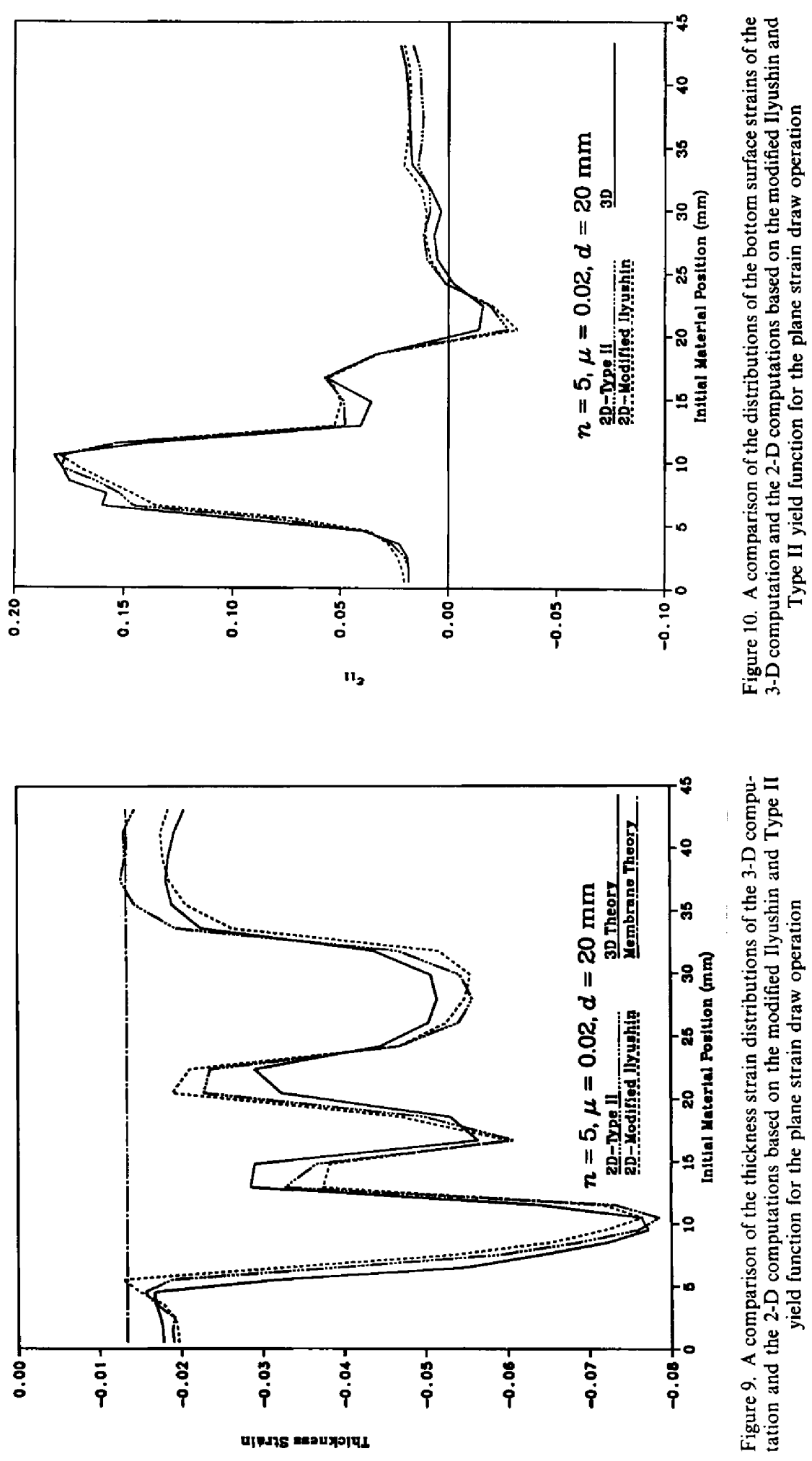


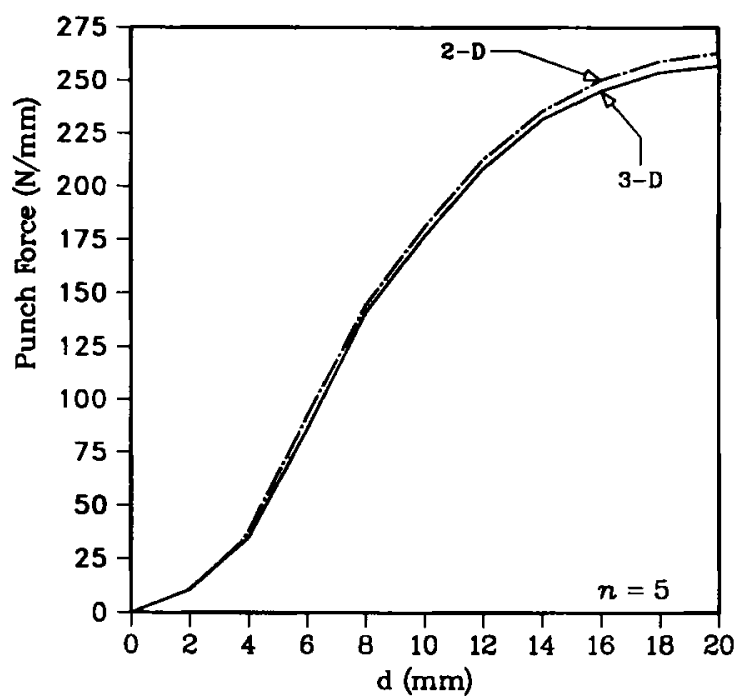

Figure 11. A comparison of the punch forces of the 3-D computation and the 2-D computation based on the Type II yield function for the plane strain drawing operation

Table III. A comparison of the results of the 3-D and 2-D computations at the punch travel distance of $20 \mathrm{~mm}$ in a plane-strain draw case. (I represents the modified Ilyushin yield function and II represents the Type II yield function.)

\begin{tabular}{lccc}
\hline Items & 2-D & $3-\mathrm{D}$ & $\begin{array}{c}\text { Error or time } \\
\text { saving (\%) }\end{array}$ \\
\hline Maximum value of & 0.0785 (I) & 0.0772 & 1.68 \\
Thickness strain & 0.0765 (II) & & 0.92 \\
Punch force & $260.81 \mathrm{~N} / \mathrm{mm}$ (I) & $256.82 \mathrm{~N} / \mathrm{mm}$ & 1.55 \\
& $262.89 \mathrm{~N} / \mathrm{mm}$ (II) & & 2.36 \\
CPU time & $73.03 \mathrm{~min}$ (I) & $174.03 \mathrm{~min}$. & 58.03 \\
& $63.99 \mathrm{~min}$ (II) & & 63.23 \\
\hline
\end{tabular}

as large as that in the hemispherical punch stretching operation. Therefore, the 2-D computations give excellent results at moderately large plastic strains. Figure 11 shows the punch forces as functions of the punch travel distances for the 3-D computation and the 2-D computation based on the Type II yield function. The results based on the modified Ilyushin and Type II yield function are virtually the same. As shown in the figure, the results of the 3-D and 2-D computations agree well.

Table III lists the results of the 3-D and the 2-D computations at the punch travel distance $d=20 \mathrm{~mm}$. When the results of the 3-D computation are used as the benchmark, the error of the maximum value of the thickness strain is less than 2 per cent, and the error of the punch force is less than 3 per cent. However, the time-saving advantage is about 60 per cent. These results indicate that the stress resultant theory can be used to simulate the elastic-plastic behaviour of plates under bending dominated conditions with the saving of computational time. 


\section{CONCLUSION}

For the first time, the results of the non-linear geometric and material behaviour of thin sheets in forming operations based on a stress resultant theory are presented in this paper. The stress resultant theory includes a quadratic yield function, a hardening rule, and the associated flow rule. This theory was employed in a finite element program to simulate a hemispherical punch stretching and a plane-strain draw operation. From the results of the simulations, we conclude that the 2-D computations based on the stress resultant theory can take account for the coupling of membrane force and bending moment and, most importantly, have a significant advantage in computational time, taking only about 40 per cent of those of the 3-D computations based on the through-the-thickness integration method. When compared to the results of the 3-D computations, the errors of the maximum radial strain and punch force are small and acceptable when the maximum strain is less than 20 per cent. Note that 20 per cent strain is well above the typical strain level in sheet forming operation for automotive outer panels.

The error of the maximum radial strain becomes large when the maximum strain is greater than 20 per cent in the hemispherical punch stretching operation. The reason is that the power-law hardening rule is too stiff at large strains when the finite deformation effects become important (Chou et al. $^{27}$ ). Therefore, the stress resultant theory generates the local phenomena such as necking later than the 3-D computation. At the final stage of the stretching operation the sheet is under stretching-dominated conditions. In general, necking starts to accelerate at the strain approximately $1 / n$ for biaxially stretched sheets depending upon the thickness variation and the loading path. We therefore expect to have the localization phenomenon when the strains become close to $1 / n$. The results of the 2-D and the 3-D computations start to deviate significant at the strain level near $1 / n$, see Figure 4 . After the hardening rule is modified, the results of the stress resultant theory are improved at large plastic strains. Therefore, the stress resultant constitutive law can be possibly used to simulate sheet metal forming processes provided that there are more comparisons of the 2-D and the 3-D computations under more complex loading as well as different punch and die geometry conditions. Further work is needed to find the theoretical basis for modification of the hardening rule at large plastic strains under combined membrane force and bending conditions when the finite deformation effects are considered.

\section{ACKNOWLEDGEMENT}

The authors acknowledge the support of this work by the National Science Foundation under grant number DDM-9102424 and the University Research Program of the Ford Motor Company.

\section{REFERENCES}

1. N. M. Wang and S. C. Tang, 'Analysis of bending effects in sheet forming operations', Int. j. numer. methods eng., 25, 253-267 (1988).

2. J. Hambrecht, S. Choudhry and J. K. Lee, 'Numerical study of two-dimensional sheet metal forming processes using bending, membrane, and solid finite element models', in E. C. Thompson et al. (eds.), Proc. Numerical Methods in Industrial Forming Processes, Fort Collins, Colorado, 1989, pp. 451-456.

3. A. S. Wifi, 'An incremental complete solution of the stretch forming and deep drawing of a circular blank using a hemispherical punch', Int. J. Mech. Sci., 18, 23-31 (1976).

4. B. S. Anderson, 'A numerical study of the deep drawing processes', in J. F. T. Pittman et al. (eds.), Proc. Numerical Methods in Industrial Forming Processes, Pineridge, Swansea, 1982, pp. 709-721.

5. A. Makinouchi, 'Elastic-plastic stress analysis of bending and hemming of sheet metal', in N.-M. Wang and S. C. Tang, (eds.), Computer Modeling of Sheet Metal Forming Process, AIME, 1985, pp. 161-176.

6. E. Oñate and O. C. Zienkiewicz, 'A viscous shell formulation for the analysis of thin sheet plate metal forming', Int. J. Mech. Sci., 25, 305-335 (1983). 
7. N. Triantafyllidis and S. K. Samanta, 'Bending effects on flow localization in metallic sheets', Proc. R. Soc. Lond., A406, 205-226 (1986).

8. N. Triantafyllidis, B. Maker and S. K. Samanta, 'An analysis of drawbeads in sheet metal forming: Part I-problem formulation', ASME J. Eng. Mater. Technol, 108, 321-327 (1986).

9. J. K. Lee, S. Choudhry, J. Hambrecht and R. H. Wagoner, 'Plane strain and axisymmetric finite element investigation of sheet metal forming processes', in Proc. Advanced Technology of Plasticity, Tokyo, Japan, 1990, pp. $1389-1396$.

10. A. A. Ilyushin, Plasticity, Gostekhizdat, Moscow, 1948. (French translation: Plasticité, Eyrolles, Paris, 1956).

11. P. G. Hodge, 'The rigid-plastic analysis of symmetrically loaded cylindrical shells', J. Appl. Mech. Trans. ASME, 21, 336-342 (1954).

12. E. T. Onat, 'The plastic collapse of cylindrical shells under axially symmetric loading', Quart. Appl. Math., 13, 63-72 (1955).

13. G. N. Brooks, 'Elastic-plastic analysis of pressurized cylindrical shells', J. Appl. Mech. ASME, 54, 597-603 (1987).

14. G. N. Brooks, 'Elastic-plastic ring-loaded cylindrical shells', J. Appl. Mech. Trans. ASME, 55, 761-766 (1988).

15. M. A. Crisfield, 'Large-deflection elasto-plastic buckling analysis of plates using finite elements', TRRL Report LR593, Transport and Road Research Laboratory, Crowthorne, Berkshire, 1973.

16. M. A. Crisfield, 'On an approximate yield criterion for thin steel shells', TRRL Report LR658, Transport and Road Research Laboratory, Crowthorne, Berkshire, 1974.

17. M. P. Bieniek and J. R. Funaro, 'Elasto-plastic behaviour of plates and shells', Report No. DNA $3954 T$, Weidlinger Associates, New York, 1976.

18. O. M. Eidsheim and P. K. Larsen, 'A study of some generalized constitutive methods for elasto-plastic shells', in W. Wunderlich, K. J. Bathe, and E. Stein, (eds.), Nonlinear Finite Element Analysis in Structural Mechanics, Springer, Berlin, 1981, pp. 364-384

19. R. S. Atkatsh, M. P. Bieniek and I. S. Sandler, 'Theory of viscoplastic shells for dynamic response', J. Appl. Mech. Trans. ASME, 50, 131-136 (1983).

20. P. Lukkunaprasit and J. M. Kelly, 'Dynamic plastic analysis using stress resultant finite element formulation', Int. J. Solids Struct., 15, 211-240 (1979).

21. L. M. Kutt and M. P. Bieniek, 'Elasto-plastic constitutive equations of stiffened plates', J. Eng. Mech., 114, 656-670 (1988).

22. L. C. Bank and M. P. Bieniek, 'Stress-resultant plasticity theories for composite laminated plates', Int. J. Plasticity, 4, 317-333 (1988).

23. P. Papadopoulos and R. L. Taylor, 'Elasto-plastic analysis of Reissner-Mindlin plates', Appl. Mech. Rev., 43, S40-S50 (1990).

24. K. N. Morman, Jr., S. C. Tang and M. D. Mathers, 'Intrinsic constitutive equations for the large deformation analysis of elastic-plastic thin shell', Technical Report SR-84-107, Ford Motor Company, 1984.

25. C. H. Chou, J. Pan, and S. C. Tang, 'Constitutive laws for thin plates of power-law materials', Int. J. Solids Struct., 27, 1387-1400 (1991).

26. C. H. Chou, J. Pan and S. C. Tang, 'A hardening rule between stress resultants and generalized plastic strains for thin plates of power-law hardening materials', J. Appl. Mech. (in press).

27. C. H. Chou, J. Pan and S. C. Tang, 'Nonproportional loading effects on elastic-plastic behaviour based on stress resultants for thin plates of strain hardening materials', Int. J. Plasticity (in press).

28. S. C. Tang, 'Large elasto-plastic strain analysis of flanged hole forming', Comput. Struct., 13, 363-370 (1981).

29. P. M. Naghdi, 'A critical review of the state of finite plasticity', J. Appl. Math. Phys. (ZAMP), 41, $315-394$ (1990).

30. B. Budiansky, 'Remarks on theories of solid and structural mechanics', Problems of Hydrodynamics and Continuum Mechanics, SlAM, Philadelphia, 1969, pp. 77-83.

31. J. W. Hutchinson, 'Finite strain analysis of elastic-plastic solids and structures', in R. F. Hartung (ed.) Numerical Solutions of Nonlinear Structural Problems, AMD, 6, ASME, New York, 1973, pp. 17-30.

32. C. H. Chou, Stress resultant constitutive laws for plates and shells of plastic materials and its applications to sheet metal forming, Ph.D. Thesis, The University of Michigan, Ann Arbor, MI, 1991. 